\title{
Neue Therapieoption für Patienten mit Knochenmetastasen
}

\begin{abstract}
- Etwa $50-70 \%$ der Tumorpatienten mit Knochenmetastasen erleiden pathologische Frakturen, schwere Knochenschmerzen oder eine Rückenmarkkompression, die in ihrer Gesamtheit als skelettbezogene Ereignisse ("skeletal-related events" = SRE) bezeichnet werden. „SRE beeinträchtigen die Betroffenen stark. Sie machen nicht nur häufig chirurgische Eingriffe oder eine Bestrahlung erforderlich, sondern können unter anderem zu einer längeren Heilungszeit, Mobilitätsverlust, verlängerten Klinikaufenthalten und einer Verkürzung der Lebenserwartung führen", berichtete Dr. Diana Lüftner, Berlin.

Bislang wurden bei Knochenmetastasen zur Prävention von SRE in der Regel Bisphosphonate eingesetzt. "Mit Denosumab (XGEVA ${ }^{\oplus}$ ) steht in dieser Indikation nun seit Kurzem ein Medikament zur Verfügung, dem ein völlig neuartiges, kausales Therapieprinzip zugrunde liegt", referierte Prof. Ingo Diel, Mannheim. Der vollständig humane, monoklonale Antikörper blockiert gezielt den RANK (Receptor Activator of NFKB)-Liganden, das zentrale Molekül für die Reifung, Aktivierung und das Überleben der Osteoklasten - und damit für die Knochenresorption.
\end{abstract}

Im Vergleich zum bisherigen Therapiestandard Zoledronsäure zeigte Denosumab in drei Phase-III-Zulassungsstudien mit mehr als 5.700 Teilnehmern eine signifikant bessere Wirksamkeit: Die Zeit bis zum Auftreten einer ersten Knochenkomplikation wurde im Mittel um 8,2 Monate verzögert ( $p<0,0001$ [Lipton A et al. Ann Oncol 2010; 21 [Suppl 8]: viii380, Abstract 1249P]). „SRE traten unter Denosumab aber nicht nur später, sondern insgesamt auch seltener auf", so Diel (relative Risikoreduktion $18 \% ; p<0,001$ ). Wie die integrierte Analyse der Zulassungsstudien zeigte, verlängerte sich unter Denosumab außerdem die Zeit bis zur Schmerzzunahme und bis zum erstmaligen Auftreten von moderaten oder starken Schmerzen um fast zwei Monate [Cleeland CS et al. Ann Oncol 2010; 21 [Suppl 8]: viii380, Abstract 1248P).

Dr. Silke Wedekind

Launch-Pressekonferenz XGEVA $^{\oplus}$ : Neue Hoffnung für Patienten mit Knochenmetastasen", 9. September 2011, München. Veranstalter: Amgen, München
Astellas unterstützt Fistula e.V.

Seit mehreren Jahren engagiert und unterstützt der Arzneimittelhersteller Astellas Pharma den deutschen Verein Fistula e.V. bei seiner Arbeit im Kampf gegen Geburtsfisteln. Etwa 100.000 Frauen leiden in Äthiopien an diesen schwerwiegenden Geburtsverletzungen. Unbehandelt sterben rund 35\% der Frauen, und wenn sie überleben, leiden sie unter Harn- und auch unter Stuhlinkontinenz.

Im Kampf gegen die Verbreitung von Geburtsfisteln wurde im Herbst 2010 mit der Eröffnung der fünften Außenklinik in Metu ein wichtiger Meilenstein erreicht. Astellas Pharma unterstützt in Metu gemeinsam mit Fistula e.V. die Ausbildung von Health Workern, die vor Ort tätig werden sollen. Diese Health Worker lernen, die Risiken für Geburtsverletzungen zu erkennen und Hilfe zu leisten.

Astellas unterstützt das Projekt auch hierzulande. Postkarten, Poster fürs Wartezimmer und Informationsbroschüren tragen dazu bei, auf das Problem der Geburtsfisteln aufmerksam zu machen. Praxen können die Informationsmaterialien kostenlos unter www.astellas.de/ fistula.html bestellen.

Nach Informationen von

Astellas, München

\section{iPhone-App „UroCongress"}

Das Unternehmen FARCO-PHARMA hat speziell für Urologen eine iPhone-App entwickelt: „UroCongress" bietet Informationen zu nationalen und internationalen urologischen Kongressen, Tagungen und Symposien. Neben elementaren Basisinformationen zu den einzelnen Veranstaltungen, wie Datum, Ort und Veranstalter, besteht auch die Möglichkeit, Veranstaltungen nach Themenschwerpunkten auszuwählen. Außerdem ist eine Funktion integriert, mit der man sich eine persönliche Favoritenliste erstellen kann. In dieser Liste lassen sich die eigenen Termine speichern und schnell wieder aufrufen. Die UroCongress-App wird kontinuierlich für das aktuelle Veranstaltungsjahr und die Folgejahre aktualisiert. Die KongressApp steht im iPhone-App-Store kostenlos zum Download zur Verfügung.

Nach Informationen von

Farco Pharma, Köln
Nach Informationen von

Olympus, Hamburg Chirurgie bekannte ICG (Indocyaningrün)- die wird demnächst ein neues Kamerasystem präsentieren. laparoskopische Standardeingriffe eignet, hat Olympus ein neues Kamerasystem zur Fluoreszenzbildgebung im PrototypenstaURO-NEWS 2011:15(10) 